\title{
Interactive comment on "Forecasting of regional methane from coal mine emissions in the Upper Silesian Coal Basin using the on-line nested global regional chemistry climate model MECO(n)(MESSy v2.53)" by Anna-Leah Nickl et al.
}

Anna-Leah Nickl et al.

anna-leah.nickl@dlr.de

Received and published: 26 February 2020

The comment was uploaded in the form of a supplement:

https://www.geosci-model-dev-discuss.net/gmd-2019-303/gmd-2019-303-AC1-

supplement.pdf 